\title{
IA et emploi : Une menace artificielle
}

\author{
Philippe Askenazy \\ CNRS-ENS-PSL, Centre Maurice Halbwachs, 48 Boulevard Jourdan 75014 Paris
}

Francis Bach

Inria, Département d'Informatique de l'Ecole normale supérieure (PSL Research University)

Résumé $^{1}$ : Les récents succès de l'IA ont nourri un discours, souvent anxiogène, d'une obsolescence du travail humain. Si des acteurs divers ont intérêt à diffuser ce message, notre analyse, nécessairement spéculative, d'informaticien et d'économiste souligne les limites technologiques et sociales de I'IA. Nous renversons ainsi la problématique : I'IA couplée aux robots sera-t-elle suffisante pour permettre aux sociétés occidentales fermées à l'immigration de répondre aux défis du XXlème siècle qui exigeront beaucoup de travail ?

\section{Introduction}

L'intelligence artificielle vit un nouvel âge d'or, où les promesses d'hier commencent à prendre corps. En effet, de nombreuses tâches où les humains excellent sont maintenant à la portée de la machine: reconnaissance d'objets dans des images, traitement du langage naturel et traduction automatique, dictées et commandes vocales, jeux de stratégie. Pour certaines d'entre elles, comme le jeu de Go, l'humain est même maintenant dépassé.

Ces progrès récents ont été en majeure partie nourris par l'utilisation systématique de nouveaux algorithmes d'apprentissage automatique (machine learning). Ceux-ci nécessitent de grandes bases de données étiquetées par des humains ainsi qu'une puissance de calcul importante, et permettent d'accomplir des tâches plus complexes que les systèmes experts précédents, dont les règles étaient en grande partie codées manuellement.

Ces performances génèrent les mêmes craintes pour l'avenir du travail et les mêmes solutions que lors du premier âge d'or de l'IA. La lecture du rapport de la National Commission on Technology, Automation and Economic Progress de 1966 au Président Lyndon Johnson est ainsi troublante. Entre autres, le diagnostic médical par les « technologies intellectuelles » est annoncé pour demain. Les auteurs dont le PDG d'IBM, James Watson, et le futur Nobel d'économie, Robert Solow, s'inquiètent alors d'un monde où, au moins transitoirement, l'ampleur des destructions d'emplois par la technologie ne pourra être compensée par des créations. Dans l'Amérique de la Guerre Froide, ils appellent donc à la mise en œuvre d'un système d'appariement informatique des emplois, à la formation des moins qualifiés et à l'introduction d'un revenu de base pour éviter une déstabilisation de la société.

\footnotetext{
${ }^{1}$ La version définitive de ce document a été publiée dans la revue Pouvoirs $N^{\circ} 170,2019$.
} 
Ces craintes sont-elles fondées maintenant que l'IA devient concrète ? Fait-on face à une mutation si profonde que la moitié des emplois doivent disparaître en quelques années dans les économies avancées ? Certes, nous défendrons que l'IA est probablement ce que les économistes nomment une technologie d'usage général, à l'instar d'Internet. Mais, l'exercice d'anticipation des impacts sur le travail ou l'emploi est hautement spéculatif tellement l'incertitude règne sur l'évolution de la technologie elle-même, son usage social ou industriel, et les mécanismes indirects qui accompagnent chaque irruption d'une telle technologie.

Chercheurs en sciences sociales d'un côté, informaticiens de l'autre, ont proposé leurs visions nécessairement diverses. Nous nous prêtons ici à l'exercice en mariant, en même temps, les réflexions d'un informaticien spécialiste du machine learning et d'un économiste spécialiste des mutations du travail.

\section{L'IA est une GPT}

Les économistes caractérisent les grandes phases technologiques par l'émergence de GPT, General Purpose Technologies, technologies à usage général. Le moteur à vapeur, l'électricité, l'automatique ou Internet font partie d'une liste qui débute en général par la domestication des plantes au Néolithique. Une GPT présente trois propriétés. Elle est utilisée dans de nombreuses activités humaines ; ses performances augmentent avec son usage ; elle facilite la construction ou l'extension d'autres innovations. L'IA couplée ou non aux robots vérifient ces trois conditions. Des usages sont déjà à l'œuvre ou programmés dans le court terme dans des secteurs aussi divers que le médical (diagnostic à partir d'imageries médicales), la finance (détection de fraudes) ou l'automobile (contrôle de la vigilance des conducteurs). Plus I'IA est utilisée, plus elle génère de données utiles qui nourrissent les processus d'apprentissage. Enfin, l'IA est déjà mobilisée par les innovateurs et les scientifiques, y compris des chercheurs en sciences sociales.

Une GPT induit trois phases pour la croissance économique. Une première est marquée sur une à trois décennies par une croissance atone de la productivité alors que la technologie est déjà visible. II s'agit d'une phase d'adaptation notamment, des régulations, des organisations du travail et des compétences, et de performances encore limitées de la technologie. On peut la qualifier de paradoxe de Solow (le Solow du rapport de 1966) en référence à son énoncé en 1987 : "on voit des ordinateurs partout sauf dans les statistiques de productivité". La phase suivante de maturité délivre une croissance de la productivité et du PIB. Dans la dernière phase, la dynamique générée par la GPT sature avant l'émergence d'une nouvelle GPT qui pourra ou non s'appuyer sur la précédente.

Nous connaissons justement depuis maintenant 10 ans dans l'ensemble des pays considérés proches de la frontière technologique, un ralentissement de la productivité du travail. Nos économies seraient donc toujours dans la première phase d'une GPT IA, et cela potentiellement pour encore plusieurs années.

Les conséquences sur le travail et l'emploi d'une GPT ont donné lieu à une très vaste littérature théorique et empirique (Voir pour une revue Ernst et al., 2018). En schématisant à la hache, des effets directs de remplacement de l'homme par la machine sur des tâches ou 
des emplois cohabitent avec des créations dans d'autres activités. Ces créations viennent de l'offre -des biens ou services nouveaux sont rendus possibles par la GPT (par exemple les réseaux sociaux pour Internet)- mais aussi du déversement de la demande : la baisse des prix induite par la GPT sur certains produits et les gains de revenus des producteurs de la GPT, permettent de consommer plus d'autres biens ou services.

Les effets de marchés sont tels que même ce schéma simple peut être mis en défaut. Ainsi les automates dans le secteur bancaire apparus au début des années 1970 devaient mécaniquement faire disparaître les emplois en agence bancaire. Le contraire est advenu : en supprimant les coûts fixes de sécurité pour créer une agence, cartes bancaires et distributeurs de billets ont attisé la concurrence entre banques, chacune cherchant à densifier son réseau d'agences physiques pour conquérir des clients. Ce n'est que près d'un demi-siècle plus tard avec le développement de la banque en ligne que l'emploi de clercs de banque a commencé à décroitre.

Au total, si une GPT implique une progressive transformation d'activités avec des " perdants " et des " gagnants » et une évolution de la formation des (futurs) actifs, elle n'emporte pas par nature une disparition du travail. II faudrait que I'IA soit d'un potentiel de remplacement qualitativement et quantitativement bien plus important que les précédentes GPT des siècles de révolutions industrielles.

Avant d'étudier ce potentiel, il est important de souligner que des acteurs divers ont intérêt à nourrir un discours "cette fois, c'est différent" au-delà de considérations purement scientifiques.

\section{Qui a intérêt au « cette fois, c'est différent »?}

Certains acteurs de la vie scientifique, politique et industrielle accompagnent cet élan de l'intelligence artificielle en soufflant sur les braises de la sur-vente ou du sensationnalisme dépourvu de fondements concrets. En effet, certains universitaires reconnus dans leur domaine mais non-experts en intelligence artificielle, comme Stephen Hawking ou Nick Bostrom, ont mis en garde contre la prise de pouvoir de la robotique ou des scénarios qui resteront de la science-fiction pour de nombreuses décennies.

L'argument que la robotique couplée à l'intelligence artificielle apportera une rupture majeure est également mobilisé par des acteurs politiques pour avancer leur agenda. Le cas du Japon de Shinzō Abe a été largement décortiqué (voir par exemple l'ouvrage de l'anthropologue Jennifer Robinson, 2018). Dès 2007 avec le plan Innovation 2025, les robots sont présentés comme une solution pour sauver la nation nippone du déclin démographique sans avoir recours à l'immigration qui menacerait son identité. Dans ce plan, les investissements massifs des entreprises et de l'Etat doivent assurer quatre promesses d'Abe. Dans la lignée des plans industriels depuis la fin du second conflit mondial, il s'agit d'assurer une suprématie technologique et une source d'exportation. Les gains de productivité dans l'industrie manufacturière comme dans les services pourvoiront aux manques de main d'œuvre. La femme libérée des tâches du foyer dont le soin des aînés et des enfants, retrouvera une appétence pour la maternité. Enfin, le robot de service 
contrairement à l'immigré peut être "nipponisé", parlant japonais lorsqu'il ne prend pas une forme humaine aux traits et tenues fantasmés de la femme et de l'homme japonais.

Le même argument de rupture est utilisé en occident, cette fois pour promouvoir le revenu de base. Comme nous l'avons signalé dans l'introduction, le revenu universel avait déjà été avancé dans les années 1960 comme remède à une disparition du travail générée par l'intelligence artificielle. Aujourd'hui des coalitions hétéroclites convergent pour promouvoir cette fable sur les deux rives de l'Atlantique. Actifs en Europe depuis les années 1980 notamment sous l'impulsion de Philippe Van Parijs, des tenants historiques du revenu universel y trouvent un nouvel argument pour leur outil censé être la version néolibérale de la protection sociale, un pendant de la flat tax pour la fiscalité. En France, la vision d'un manque structurel d'emplois induits par l'IA et les robots est portée également à gauche par des (ex-)socialistes ou des écologistes. La promesse est là d'une forme de libération de l'homme de la contrainte de travailler ou de l'exploitation. Cette émancipation serait assurée par une taxe sur les robots qui aiderait à financer un revenu universel décent. Outre Atlantique, on retrouve pèle mêle des démocrates, des libertariens et des milliardaires de la Silicon Valley (Elon Musk, Mark Zuckerberg,...) pour défendre le couple IA-robots/revenu de base. Chacun y voit une cohérence avec son agenda, respectivement, revivifier la protection sociale, remplacer les dispositifs sociaux ciblés et éviter une remise en cause de l'ordre capitaliste qu'ils dominent.

Enfin, l'intelligence artificielle étant devenue un enjeu de domination industrielle, certaines entreprises déjà très puissantes, en particulier les "GAFAM" (Google, Apple, Facebook, Amazon, Microsoft), sur-vendent le plus souvent les performances réelles de leurs algorithmes en insistant par exemple sur le surpassement de la performance humaine. Ces entreprises communiquent par contre moins sur les problèmes de protection de la vie privée que pose la collecte massive des données personnelles qui nourrissent le développement de leurs propres produits. Enfin, les bénéfices potentiels de la mise en oeuvre de l'IA dans les entreprises constituent un argument de vente pour bon nombre de start-ups et cabinets de consultants.

\section{Quel travail I'IA fait, saura faire, ou ne pourra pas faire?}

Que sait déjà accomplir l'intelligence artificielle? L'état de l'art actuel est presque intégralement obtenu par apprentissage: on est passé d'une mise en équation explicite des raisonnements humains dans des systèmes à base de règles, au développement de grands jeux de données que des humains ont dû étiqueter manuellement. Par exemple, des millions d'images annotées avec leur contenu (chien, chat, piéton, visages, voiture, vélo, etc.) sont nécessaires pour la reconnaissance visuelle ; des millions d'heures de parole avec leurs retranscriptions écrites sont utilisées pour la dictée vocale des téléphones portables modernes. Ces données sont ensuite traitées par un algorithme d'apprentissage automatique, dont le résultat est lui-même un algorithme qui pourra reconnaître les mêmes classes d'objets (chien, chat, etc.), mais dans des images nouvelles jamais observées auparavant. Cet étiquetage actuellement indispensable pour les technologies actuelles est laborieux et compte parmi les nouveaux emplois peu rémunérés et souvent délocalisés que l'intelligence artificielle et plus généralement les nouvelles technologies numériques ont engendrés (voir l'étude approfondie d'Antonio A. Casilli, 2019). Pour certaines tâches spécifiques comme les jeux (Go, échecs), l'ordinateur peut simuler des parties à l'infini et 
ainsi acquérir lui-même automatiquement les données nécessaires, mais cela nécessite de pouvoir simuler parfaitement l'environnement où agira l'algorithme d'intelligence artificielle.

Au-delà de ce besoin de données, la dépense énergétique des ordinateurs nécessaires aux algorithmes d'IA croît rapidement; par exemple, les expériences de certains articles de recherche récents en apprentissage automatique correspondent à la consommation électrique d'un foyer Français pendant cent ans. Cette gourmandise préoccupante en énergie compte d'ailleurs parmi les secrets les mieux gardés par les grands groupes industriels.

Par nature, les méthodes actuelles de l'IA ne peuvent se mettre en œuvre que sur des tâches pré-définies (comme reconnaître un piéton dans une image ou jouer au jeu de Go): si les méthodes d'apprentissage utilisées sont génériques, les algorithmes finaux et les données sont spécifiques à chacune des tâches. Nous sommes encore loin d'une intelligence "multi-usage" qui permet de s'adapter à des situations qui n'ont jamais été observées auparavant (l'algorithme qui a battu le champion du monde de Go ne pourra pas être utilisé directement pour jouer aux échecs). De plus, ces tâches doivent souvent être déjà sous forme numérique, même si les progrès constants de la robotique laissent entrevoir l'utilisation de robots dans des situations plus incertaines et moins contrôlées que des ateliers d'usine. Ainsi, les technologies actuelles permettent d'aider les humains dans certaines tâches (par exemple, la recherche d'information, ou la traduction automatique d'une langue à une autre), sans pour autant pouvoir les remplacer.

Certaines applications concrètes récentes ont déjà eu un fort impact sociétal et économique, avec une modification des marchés sous-jacents, quelques fois une création d'activités nouvelles, mais pas de pertes massives d'emplois : le ciblage de la publicité, en particulier sur internet, est effectué par des algorithmes qui tracent le parcours des utilisateurs; par ailleurs, les contenus proposés aux clients des réseaux sociaux ou aux lecteurs de fils d'actualités sont personnalisés et choisis par des algorithmes d'intelligence artificielle. Mais comment aller plus loin?

C'est un défi à la fois technique et scientifique pour les prochaines décennies. De nombreuses applications dont on parle déjà, comme la voiture autonome ou la médecine personnalisée, ne seront possibles que si certains verrous sont levés, comme le manque de robustesse aux situations non observées et aux données lacunaires: par exemple, pour la voiture autonome, si un algorithme de reconnaissance visuelle de l'environnement immédiat du véhicule a été entraîné sur des données acquises à Nice en été où la météo est ensoleillée, la reconnaissance sera moins performante à Nancy lors d'une averse de neige; il faut donc avoir observé toutes les situations possibles. Ainsi de nouvelles avancées scientifiques sont nécessaires pour réduire la gourmandise en données étiquetées et l'empreinte énergétique. De plus, une fois déployés dans le monde réel au-delà des laboratoires et des simulations numériques, les algorithmes d'IA devront pouvoir évaluer l'incertitude de leurs prédictions. Enfin, dans les applications critiques où la sécurité ou la santé sont en jeu, ces algorithmes devront pouvoir justifier leurs décisions auprès des utilisateurs, des organismes de certification, et le cas échéant du législateur et du juge. Même pour les algorithmes les plus récents, la technologie actuelle ne le permet pas encore. D'autant que les informations erronées des capteurs trompent aujourd'hui les 
systèmes experts beaucoup plus simples avec des conséquences parfois dramatiques vues par exemple avec les crashs de Boeing 737 Max.

Or en l'absence d'explicabilité, outre les questionnements éthiques, se pose l'acceptabilité des décisions par les consommateurs, les patients ou les travailleurs ; la méfiance sera d'autant plus prégnante si l'Etat oublie d'offrir à tous une éducation comportant une acculturation suffisante aux sciences notamment l'informatique et les mathématiques ${ }^{2}$. Et comment éviter des décisions conservatrices, qui par exemple dans le domaine du recrutement reproduiraient des biais discriminatoires de genre ou d'ethnicité ?

Au-delà des erreurs des capteurs automatiques, l'IA peut également être mise en défaut par la manipulation humaine des données : les sciences de gestions montrent ainsi que les salariés et managers ont appris à s'adapter aux outils quantitatifs de gestion comme les progiciels de gestion intégrés, en rentrant des données qui satisferont la machine.

Un autre obstacle cette fois économique est la dimension propriétaire des données. Ainsi, des véhicules autonomes nécessiteraient un vaste partage des données entre les véhicules et leurs environnements (téléphone mobile des passants...), via notamment l'internet des objets; les GAFAM accepteront-ils un tel partage qui permettrait aux constructeurs automobiles de devenir des concurrents dans le domaine des données?

\section{Une solution au trop plein de travail ?}

Même si l'on admet que les obstacles technologiques ou sociétaux seront rapidement levés, un regard sur l'état actuel des marchés du travail permet d'apprécier combien une pénurie de travail est lointaine.

Le mouvement historique de réduction de la durée annuelle de travail s'est arrêté depuis le début du siècle dans les pays avancés. Les années de vie au travail augmentent dans le sillage des réformes des systèmes de pensions. Avec un taux d'emploi actuel -part des personnes en emploi parmi celles en âge de travailler- similaire à celui des années 1990 et début 2000, les Etats-Unis font figure d'exception. Le taux d'emploi des 15-64 ans atteint des records dans de nombreux pays, non seulement ceux en hiver démographique, Japon ou Allemagne, mais aussi parmi ceux connaissant encore une croissance de la population comme le Royaume-Uni. La France souffre toujours certes d'un fort taux de chômage, mais le nombre d'emplois a fortement progressé et même le taux d'emplois : selon les données de l'INSEE, le taux d'emploi des 15-64 ans a atteint $65.4 \%$ en 2018 contre seulement $60.7 \%$ en 1995 au moment de l'émergence d'Internet. L'Espagne et l'Italie n'ont certes pas encore retrouvé leur niveaux d'avant la Grande Récession, mais le taux d'emploi y est plus de 7 à 10 points supérieur à celui de la fin du siècle. Parallèlement, les diverses enquêtes sur les conditions de travail montrent une intensification du travail pour la plupart des catégories professionnelles.

\footnotetext{
${ }^{2}$ C'est le cas aujourd'hui en France avec la réforme du baccalauréat général. Les mathématiques deviennent une spécialité facultative dès la première. Un tronc commun de sciences se contentera de survoler en deux heures hebdomadaires sciences physiques, sciences de la vie et de la terre, et mathématiques. Il ne comporte pas explicitement d'informatique, de numérique ni de sciences de l'ingénieur.
} 
S'il y avait un " cette fois, c'est différent ", il se trouverait dans des réservoirs plus limités de main d'œuvre que lors des précédentes vagues de GPT : aucun secteur ne peut libérer autant de travailleurs que ce que permit la modernisation de l'agriculture, la participation féminine a déjà fortement augmenté, l'espérance de vie en bonne santé stagne autour de 65 ans, la politique migratoire est très restrictive, et la démographie naturelle est en berne.

Or deux enjeux majeurs pour nos sociétés vont imposer des besoins de main d'œuvre massifs : la dépendance et l'adaptation au changement climatique. Si les exosquelettes ou les robots d'interaction peuvent augmenter la productivité d'un personnel de soin ou d'un ouvrier chargé de l'isolation d'un logement ancien, nos analyses des capacités de l'IA et des robots, ne permettent pas d'envisager dans les décennies où vont émerger ces besoins, que ces technologies pourront pourvoir les tâches nécessaires dans ces activités. Les premières études sur les établissements médicalisés japonais utilisateurs de robots le confirment.

Ainsi, nous proposons de renverser totalement la perspective sur l'IA couplée aux robots : ces technologies à usage général pourront-elles suffisamment engendrer des gains de productivité et libérer des travailleurs dans certaines activités pour se déverser vers d'autres à revaloriser ? Permettront-elles aux sociétés occidentales fermées à l'immigration de répondre aux défis du XXlème siècle?

\section{Références}

Casilli, Antonio (2019). En attendant les robots. Enquête sur le travail du clic. Editions du Seuil.

Ernst, Ekkehard \& Merola, Rossana \& Samaan, Daniel. (2018). "The economics of artificial intelligence: Implications for the future of work", ILO future of work Research paper series, $\mathrm{N}^{\circ} 5$.

Robertson, Jennifer. (2018). Robo sapiens japanicus: Robots, Gender, Family, and the Japanese Nation. Oakland: University of California Press.

Renouveau de l'Intelligence Artificielle et de l'Apprentissage Automatique (Mars 2018). Rapport de l'Académie des technologies. 